\title{
Mitophagy in the Skeletal Muscle Is Suppressed in Spleen Qi Deficiency
}

\author{
Dan Ma1, Wenjun Liu², Lu Wang1, Xinzhu Xu${ }^{1}$, Lingzhi Wang1, Huaxin Yu1, Xudong Liu1, \\ Huihui Liu', Deshan Wang1, Dehong Shan ${ }^{*}$ \\ ${ }^{1}$ School of Basic Medicine, Liaoning University of Traditional Chinese Medicine, Shenyang, China \\ ${ }^{2}$ Teaching and Experimental Center, Liaoning University of Traditional Chinese Medicine, Shenyang, China \\ Email: *dehongshan@163.com
}

How to cite this paper: Ma, D., Liu, W.J., Wang, L., Xu, X.Z., Wang, L.Z., Yu, H.X., Liu, X.D., Liu, H.H., Wang, D.S. and Shan, D.H. (2019) Mitophagy in the Skeletal Muscle Is Suppressed in Spleen Qi Deficiency. Chinese Medicine, 10, 11-18. https://doi.org/10.4236/cm.2019.101002

Received: December 27, 2018

Accepted: January 27, 2019

Published: January 30, 2019

Copyright $\odot 2019$ by author(s) and Scientific Research Publishing Inc. This work is licensed under the Creative Commons Attribution International License (CC BY 4.0). http://creativecommons.org/licenses/by/4.0/

\begin{abstract}
Objective: Mitochondrial impairment in the skeletal muscle contributes to useless of limbs in spleen qi deficiency; however the genesis of such impairment is not clear. Herein, PTEN-induced putative kinase 1 (PINK1)-Parkin pathway and mitophagy were studied to explore the machinery of mitochondrial impairment. Methods: 16 male SD rats were randomly divided in the control group and spleen qi deficiency group (model group); transmission electron microscope was used to observe mitochondrial morphology; mitochondrial oxidative phosphorylation was assessed by testing mitochondrial membrane potential (MMP) and levels of ATP and ROS; western blot was used to analyze expressions of PINK1, Parkin, microtubule-associated protein 1 light chain 3-II (LC3-II) and p62. Results: Compared with those in the control group, mitochondria became small, less and scattered, MMP and the ATP level were reduced, the ROS level was elevated, PINK1 expression was decreased, p62 expression was increased, but Parkin and LC3-II expressions were not altered, in the model group. Conclusions: Suppression of mitophagy might be related to the mitochondrial damage in the skeletal muscle when spleen qi deficiency develops.
\end{abstract}

\section{Keywords}

Spleen Qi Deficiency, Mitophagy, PINK1, Parkin, LC3, p62, Muscle

\section{Introduction}

According to the theory of the traditional Chinese medicine, spleen can transform and transport cereal nutrients to muscles; hence spleen dominates muscles and limbs. Actually, such a function of spleen relies greatly on the conditions of 
spleen qi. When spleen qi is vigorous, muscles are healthy and strong, while spleen qi deficiency will lead to useless of limbs.

In the modern medicine, muscular contraction is adenosine triphosphate (ATP)-dependent interaction of filaments; therefore mitochondrial health affects muscular strength. The mitochondrion is the cellular powerhouse, generating ATP via oxidative phosphorylation (OXPHOS). In addition, mitochondrial membrane potential (MMP) and reactive oxidative species (ROS) are byproducts of OXPHOS, and their normality is helpful for ATP production and cellular survival [1] [2]. However, stress damages mitochondria easily, resulting in MMP dissipation and ROS accumulation which impair cellular components and induce oxidative stress and apoptosis [3] [4]. Generally, MMP dissipation can activate PTEN-induced putative kinase 1 (PINK1)-Parkin pathway to initiate mitophagy by which cells degrade abnormal mitochondria in lysosomes to maintain mitochondrial health [5] [6].

It is thought that mitochondrial impairment in the skeletal muscles contributes to useless of limbs [7], but the pathogenesis of such impairment is not clear yet. In the present study, mitochondrial morphology and OXPHOS, PINK1-Parkin pathway and mitophagy-related protein expressions in the skeletal muscle were investigated respectively, attempting to explore the mechanism of mitochondrial impairment in spleen qi deficiency.

\section{Materials and Methods}

\subsection{Animals}

Sixteen male SD rats $(220 \pm 10 \mathrm{~g})$ (Liaoning Changsheng Biotechnology Co., Ltd (SCXK (Liao) 2010-0001) were habituated for one week under the condition of temperature $\left(22^{\circ} \mathrm{C} \pm 2^{\circ} \mathrm{C}\right)$, humidity $(55 \% \pm 5 \%)$ and a light/dark cycle (light on at 8:00 and off at 20:00), and then randomly divided into the control group and spleen qi deficiency model group (model group) of 8 each. Animal care procedures were carried out in accordance with the National Institutes of Health Guide for the Care and Use of Laboratory Animals, and every effort was made to minimize the animal suffering.

\subsection{Establishment and Evaluation of the Spleen Qi Deficiency Model}

Spleen qi deficiency model was established and assessed according to our previous study [8]. Briefly, in 2 weeks, rats in the model group were provided with feed and water ad libitum on odd days and fed only with cabbage on even days, meanwhile they were forced to swim to fatigue in $35^{\circ} \mathrm{C}-37^{\circ} \mathrm{C}$ water every day. Evaluation standard included emaciation, poor appetite, mental and physical fatigue.

\subsection{Mitochondrial Morphology}

Rats were anesthetized with $10 \%$ chloral hydrate $(0.35 \mathrm{~mL} / 100 \mathrm{~g})$ and sacrificed 
by decapitation. The quadriceps femoris was taken and cut into pieces of $1 \mathrm{~mm}^{3}$ at $4^{\circ} \mathrm{C}$ and fixed with $2.5 \%$ glutaraldehyde in phosphate buffer. After dehydration in ethanol with graded concentrations, specimens were treated with propyleneoxide and embedded in Epon. Ultrathin sections $(60 \mathrm{~nm})$ stained with uranyl acetate and lead citrate were examined in a transmission electron microscope (JEM-1 200EX; Jeol, Tokyo, Japan) at $120 \mathrm{kV}$.

\subsection{MMP Assay}

$200 \mathrm{mg}$ tissue sample from the quadriceps femoris was homogenized in $500 \mu \mathrm{L}$ mitochondrial isolation buffer $(220 \mathrm{mM}$ Mannitol, $70 \mathrm{mM}$ Sucrose, $10 \mathrm{mM}$ Tris-base and $1 \mathrm{mM}$ EDTA, pH 7.4) on ice. After homogenates were centrifuged $\left(1000 \mathrm{~g}, 5 \mathrm{~min}, 4^{\circ} \mathrm{C}\right.$ ), the supernatant was transferred into a new microcentrifuge tube and centrifuged again at the same condition, and then the supernatant was draw and centrifuged $\left(12,000 \mathrm{~g}, 10 \mathrm{~min}, 4^{\circ} \mathrm{C}\right)$. Finally, the supernatant was removed and the pellet was saved in $100 \mu \mathrm{L}$ PBS ( $\mathrm{pH}$ 7.4). MMP was determined using a JC-1 MMP Assay Kit (Beijing solarbio science and technology co., ltd., Beijing, China) according to the instructions of the manufacturer. MMP in the model group was expressed in percent of the control group that was set as $100 \%$.

\subsection{ATP and ROS Assay}

The ATP level in the muscular tissue was detected by a ATP Bioluminescence Assay kit (Beyotime Institute of Biotechnology, Haimen, Jiangsu, China) according to the protocol of the manufacturer. The level of hydrogen peroxide was used to reflect the ROS level and measured using a kit following the instructions of the manufacturer (Beyotime Institute of Biotechnology, Haimen, Jiangsu, China). The level of ROS of the model group was expressed in percent of the control group.

\subsection{Western Blot}

The total cellular proteins of muscular tissues were extracted by lysis of cells with buffer containing $150 \mathrm{mM} \mathrm{NaCl}, 0.1 \%$ Triton X-100, 0.5\% deoxycholate, $0.1 \%$ sodium dodecyl sulfate, $50 \mathrm{mM}$ Tris- $\mathrm{HCl}(\mathrm{pH}$ 7.0) and $1 \mathrm{mM}$ ethylenediaminetetraacetic acid (EDTA). Protein concentrations were determined using the bicinchoninic acid method. Equal amounts of protein $(50 \mu \mathrm{g})$ were subjected to sodium dodecyl sulfate-polyacrylamide gel electrophoresis on $10 \%$ gels and transferred to a polyvinylidene difluoride membrane. The membrane was blocked for $1 \mathrm{~h}$ with blocking buffer containing 5\% non-fat dry milk and 0.05\% Tween-20 in Tris-buffered saline, followed by sixteen hours incubation at $4^{\circ} \mathrm{C}$ with the primary antibodies as follows: PINK1 (1:1000; Abcam), Parkin (1:1000; Proteintech), LC3-II (1:2000; Abcam) and p62 (1:2000; Proteintech). Following further incubation with the corresponding secondary antibody (1:5000; Transgen, China) for $1 \mathrm{~h}$ at room temperature, immune complexes were detected using enhanced chemiluminescence western blotting reagents (TransGen, China). 
Images were quantified by AlphaView (Version: 3.4.0.0) (ProteinSimple, USA). GAPDH was served as the internal standard. The protein expression in the model groups was expressed in the percent of the control group.

\subsection{Statistical Analysis}

The data were expressed as mean \pm S.D., and statistical analysis was performed between groups by ANOVA using SPSS 16.0 software (SPSS Inc., Chicago, IL, USA). A value of $P<0.05$ or less was considered statistical significance.

\section{Results}

\subsection{Assessment of Spleen Qi Deficiency}

Table 1 showed that 4 items of the model group were all lesser than those of the control group. And among them, reduced body mass and food intake implied emaciation and poor appetite respectively, decreased motion distance and vertical times in the open field implied mental fatigue, and lessened grasping strength represented physical fatigue. Therefore, these data suggested the establishment of spleen qi deficiency in rats.

\subsection{Mitochondrial Morphology}

Figure 1 showed that mitochondria were between myofibrils exhibiting clear Z and $\mathrm{M}$ lines, and dark and light bands. Mitochondria in the control group were rod-shape and abundant of cristae (Figure 1(A)), while those in the model group were small, less and scattered, but their cristae were still abundant (Figure $1(B))$.

\subsection{Assessment of Mitochondrial OXPHOS}

Compared with those in the control group, MMP and the ATP level were reduced whereas the ROS level was increased, significantly, in the model group (Table 2).

Table 1. Model evaluation of rat spleen qi deficiency.

\begin{tabular}{cccccc}
\hline & $\begin{array}{c}\text { Body mass } \\
(\mathrm{g})\end{array}$ & $\begin{array}{c}\text { Food intake } \\
(\mathrm{g})\end{array}$ & $\begin{array}{c}\text { Motion distance } \\
(\mathrm{cm})\end{array}$ & $\begin{array}{c}\text { Vertical } \\
\text { times }\end{array}$ & $\begin{array}{c}\text { Grasping strength } \\
(\mathrm{g})\end{array}$ \\
\hline $\begin{array}{c}\text { Control } \\
\text { group }\end{array}$ & $305.40 \pm 10.81$ & $31.20 \pm 4.08$ & $8611.47 \pm 984.55$ & $18.5 \pm 3.7$ & $1614.70 \pm 316.82$ \\
$\begin{array}{l}\text { Model } \\
\text { group }\end{array}$ & $192.60 \pm 8.35^{\star *}$ & $21.45 \pm 4.70^{*}$ & $5874.53 \pm 428.70^{\star *}$ & $12.7 \pm 3.2^{*}$ & $1117.07 \pm 144.40^{* *}$ \\
\hline
\end{tabular}

Values are means \pm S.D. $(\mathrm{n}=8) .{ }^{*} P<0.05,{ }^{* *} P<0.01$ versus the control.

Table 2. Assessment of the mitochondrial OXPHOS in two groups.

\begin{tabular}{cccc}
\hline & MMP $(\%)$ & ATP $(\mu \mathrm{mol} / \mathrm{g})$ & ROS $(\%)$ \\
\hline Control group & 100 & $0.404 \pm 0.180$ & 100 \\
Model group & $58.21 \pm 9.95^{* *}$ & $0.213 \pm 0.086^{* *}$ & $117.58 \pm 9.49^{*}$ \\
\hline
\end{tabular}

Values are means \pm S.D. $(\mathrm{n}=8) .{ }^{\star} P<0.05,{ }^{* *} P<0.01$ versus the control. 

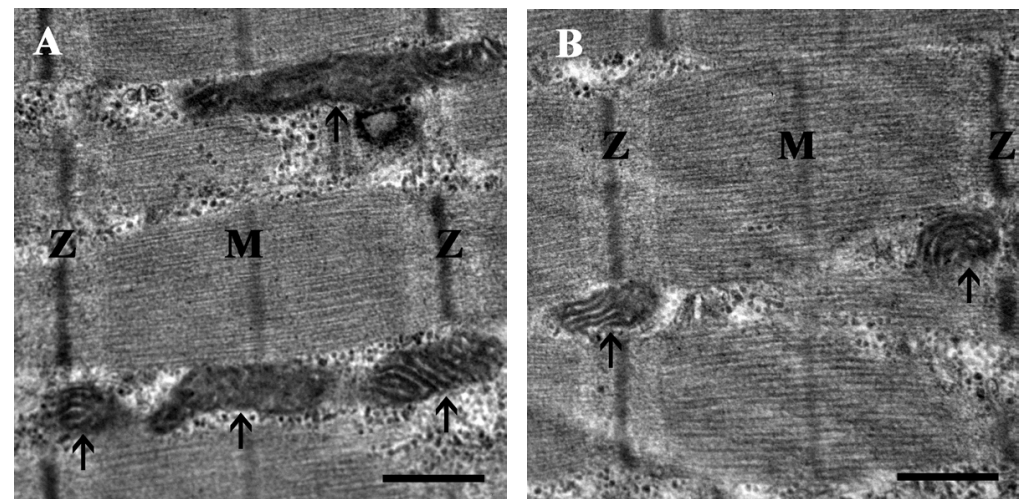

Figure 1. Mitochondrial morphology in the skeletal muscle fiber. (A) The control group; (B) The model group. Arrow: Mitochondrion; M: M line; Z: $\mathrm{Z}$ line. Scale bar, $500 \mathrm{~nm}$. Magnification: 25,000×.

\subsection{Expressions of PINK1, Parkin, LC3-II and p62}

Relative expressions PINK1, Parkin, LC3-II and p62 of the model group to the control group were $60.55 \% \pm 8.42 \%, 88.41 \% \pm 5.00 \%, 88.66 \% \pm 1.41 \%$ and $119.31 \% \pm 3.35 \%$, and PINK1 expression was decreased whereas p62 expression was increased significantly ( $\mathrm{n}=3, P_{\mathrm{s}}<0.01$ ) (Figure 2 ).

\section{Discussion}

In the traditional Chinese medicine, muscles and limbs are dominated by spleen because spleen can transform and transport nutrients to nourish them. Therefore, patients with spleen qi deficiency often complain of useless of limbs. It has reported that mitochondrial impairment might contribute to useless of limbs [7], but the genesis of mitochondrial impairment is not clear.

Herein, mitochondrial morphology and function in the skeletal muscles were investigated respectively. First, it was observed that mitochondria in the model group became small, less and scattered, suggesting the morphological damage. Secondly, compared with those in the control group, the ATP level and MMP were lower whereas the ROS level was higher in the model group, indicating functional abnormality. Taken together, mitochondria in the skeletal muscle were impaired after spleen qi deficiency occurred.

Mitochondria not only generate energy for cells but also induce oxidative stress and apoptosis if ROS accumulation; hence cells develop multiple mechanisms to maintain the mitochondrial OXPHOS [9]. OXPHOS within mitochondria is an important metabolic process whereby electrons are transferred through the electron transport chain across the inner mitochondrial membrane to reduce oxygen into water. ATP is the key product of OXPHOS and its significant reduction inhibits cellular activities including decreasing muscular strength. ROS are highly reactive byproducts of mitochondrial respiration and their accumulation will impair cellular components and activate death pathways; however, cells initiate related mechanisms such as mitophagy to opposite ROS accumulation [10] [11] [12]. MMP is another byproduct of OXPHOS, and its 


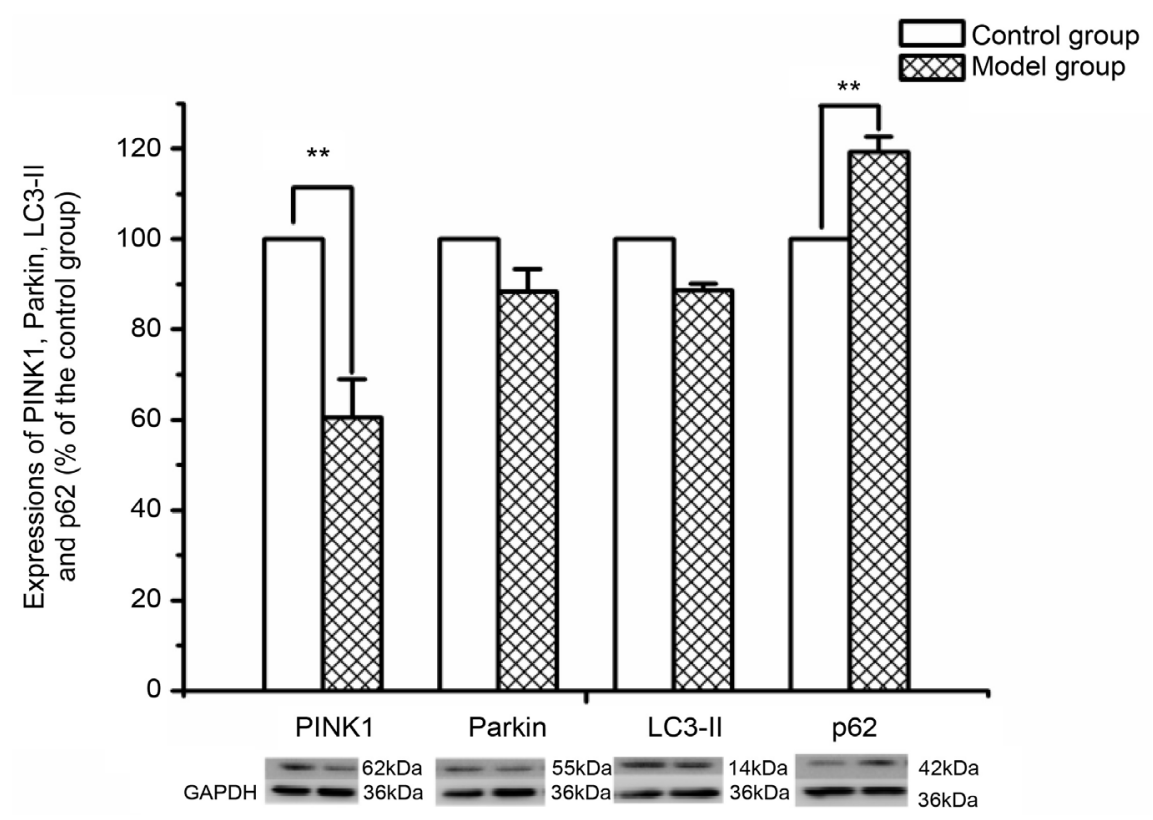

Figure 2. Relative expressions of PINK1, Parkin, LC3-II and p62 in two group. Values are means \pm S.D. $(\mathrm{n}=3) .{ }^{*} P<0.01$ versus the control.

reduction can activate PINK1-Parkin pathway to induce mitophagy [13] [14].

Mitophagy is a special autophagy by which cells destroy abnormal mitochondria. PINK1-Parkin pathway is one of the best well-known pathways mediating mitophagy. It is thought that normal mitochondria can import PINK1 into mitochondrial matrix and degrade it; however, mitochondria exhibiting lower MMP cannot import PINK1 but induce PINK1 accumulation on the outer mitochondrial membrane (OMM), and then phosphorylates ubiquitin, leading to the recruitment of cytosolic Parkin and subsequently the engulfment of depolarized mitochondria by autophagosomes [15] [16]. In the present study, PINK1 expression was significantly reduced while Parkin expression was unaltered in the model group, suggesting that PINK1 might not accumulate on OMM and Parkin recruitment was inhibited after spleen qi deficiency developed. As PINK1-Parkin pathway in the muscle fiber was suppressed in spleen qi deficiency, it was speculated that mitophagy might be not promoted.

As mitophagy is tightly controlled by a number of proteins, some proteins are used to assess mitophagy. LC3-II, a widely used marker of autophagy, is localized at autophagosomes and its expression level correlates with the number of autophagosomes [17] [18]. Another widely used autophagy marker is p62, also called sequestosome 1 (SQSTM1), which directly binds to LC3 and is selectively degraded by autophagy [19] [20]. In the present study, it was found that LC3-II expression was unchanged while p62 expression was elevated in the model group. Such results indicated that mitophagy in the skeletal muscle fiber might be inhibited in spleen qi deficiency.

In conclusion, suppression of mitophagy in the skeletal muscles might be related to the mitochondrial impairment in spleen qi deficiency. 


\section{Funding}

This study was supported by National Natural Science Foundation of China (81673851, 81803986) and the Open fund of Key Laboratory of Ministry of Education for TCM Viscera-State Theory and Applications, Liaoning University of Traditional Chinese Medicine (zyzx1601).

\section{Conflicts of Interest}

The authors report no conflict of interest.

\section{References}

[1] Wilson, D.F. (2017) Oxidative Phosphorylation: Regulation and Role in Cellular and Tissue Metabolism. The Journal of Physiology, 595, 7023-7038.

https://doi.org/10.1113/JP273839

[2] Zakharova, V.V., Pletjushkina, O.Y., Zinovkin, R.A., Popova, E.N. and Chernyak, B.V. (2017) Mitochondria-Targeted Antioxidants and Uncouplers of Oxidative Phosphorylation in Treatment of the Systemic Inflammatory Response Syndrome (SIRS). Journal of Cellular Physiology, 232, 904-912.

https://doi.org/10.1002/jcp.25626

[3] Broome, S.C., Woodhead, J.S.T. and Merry, T.L. (2018) Mitochondria-Targeted Antioxidants and Skeletal Muscle Function. Antioxidants (Basel), 7, pii: E107. https://doi.org/10.3390/antiox7080107

[4] Evans, C.S. (2018) Autophagy and Mitophagy in ALS. Neurobiology of Disease, Available Online.

[5] McWilliams T.G. (2017) PINK1 and Parkin: Emerging Themes in Mitochondrial Homeostasis. Current Opinion in Cell Biology, 45, 83-91. https://doi.org/10.1016/j.ceb.2017.03.013

[6] Cummins, N. (2018) Shedding Light on Mitophagy in Neurons: What Is the Evidence for PINK1/Parkin Mitophagy In Vivo? Cellular and Molecular Life Sciences, 75, 1151-1162. https://doi.org/10.1007/s00018-017-2692-9

[7] Liu, Y., Wang, C., Liu, J., Zhou, J., Liu, Z., Ou, Z., et al. (2006) Si-Jun-Zi Decoction Repairs Mitochondrial Damage of Cells of Liver, Myocardium, Gastric Mucosa and Skeletal Muscle in Rats with Spleen Asthenia. Chinese Journal of Clinical Rehabilitation, 39, 170-173+193.

[8] Zhao, F., Liu, X., Liu, W., Cai, J., Wang, L., Yu, H., et al. (2014) Expression Imbalance of Cholinergic M2 and M3 Receptors Contributes to the Motility Reduction of the Small Intestine in Spleen Qi Deficiency. Chinese Medicine, 5, 4. https://doi.org/10.1186/1749-8546-5-4

[9] Grgic, J., Schoenfeld, B.J., Skrepnik, M., Davies, T.B. and Mikulic, P. (2018) Effects of Rest Interval Duration in Resistance Training on Measures of Muscular Strength: A Systematic Review. Sports Medicine, 48, 137-151. https://doi.org/10.1007/s40279-017-0788-x

[10] Jaiswal, N., Maurya, C.K., Arha D., Avisetti, D.R., Prathapan, A., Raj, P.S., et al. (2015) Fructose Induces Mitochondrial Dysfunction and Triggers Apoptosis in Skeletal Muscle Cells by Provoking Oxidative Stress. Apoptosis, 20, 930-947. https://doi.org/10.1007/s10495-015-1128-y

[11] Fiorenza, M., Gunnarsson, T.P. and Ehlers, T.S. (2018) High-Intensity Exercise Training Ameliorates Aberrant Expression of Markers of Mitochondrial Turnover 
But Not Oxidative Damage in Skeletal Muscle of Men with Essential Hypertension. Acta Physiologica, e13208. https://doi.org/10.1111/apha.13208

[12] Yan, C. and Li, T.S. (2018) Dual Role of Mitophagy in Cancer Drug Resistance. Anticancer Research, 38, 617-621.

[13] Song, L., Huang, Y., Hou, X., Yang, Y., Kala, S., Qiu, Z., et al. (2018) PINK1/Parkin-Mediated Mitophagy Promotes Resistance to Sonodynamic Therapy. Cellular Physiology and Biochemistry, 49, 1825-1839. https://doi.org/10.1159/000493629

[14] Peker, N., Donipadi, V., Sharma, M., McFarlane, C. and Kambadur, R. (2018) Loss of Parkin Impairs Mitochondrial Function and Leads to Muscle Atrophy. American Journal of Physiology-Cell Physiology, 315, C164-C185. https://doi.org/10.1152/ajpcell.00064.2017

[15] Greene, A.W., Grenier, K., Aguileta, M.A., Muise, S., Farazifard, R., Haque, M.E., et al. (2012) Mitochondrial Processing Peptidase Regulates PINK1 Processing, Import and Parkin Recruitment. EMBO Report, 13, 378-385.

https://doi.org/10.1038/embor.2012.14

[16] Jin, S.M., Lazarou, M., Wang, C., Kane, L.A., Narendra, D.P. and Youle, R.J. (2010) Mitochondrial Membrane Potential Regulates PINK1 Import and Proteolytic Destabilization by PARL. The Journal of Cell Biology, 191, 933-942. https://doi.org/10.1083/jcb.201008084

[17] Farkas, T. (2017) Renilla Luciferase-LC3 Based Reporter Assay for Measuring Autophagic Flux. Methods in Enzymology, 588, 1-13.

[18] Oh, S.H., Choi, Y.B., Kim, J.H., Weihl, C.C. and Ju, J.S. (2017) Quantification of Autophagy Flux Using LC3 ELISA. Analytical Biochemistry, 530, 57-67. https://doi.org/10.1016/j.ab.2017.05.003

[19] Jiang, P. and Mizushima, N. (2015) LC3- and p62-Based Biochemical Methods for the Analysis of Autophagy Progression in Mammalian Cells. Methods, 75, 13-18. https://doi.org/10.1016/j.ymeth.2014.11.021

[20] Morgan, M.J. and Thorburn, A. (2016) Measuring Autophagy in the Context of Cancer. Advances in Experimental Medicine and Biology, 899, 121-143. https://doi.org/10.1007/978-3-319-26666-4_8 